\title{
Clinical applications of patient-specific induced pluripotent stem cells in cardiovascular medicine
}

\author{
Yingzi Oh, ${ }^{1}$ Heming Wei, ${ }^{2}$ Dongrui Ma, ${ }^{2}$ Xiaoming Sun, ${ }^{2}$ Reginald Liew ${ }^{1,2}$
}

'Duke-NUS Graduate Medical School, Singapore, Singapore ${ }^{2}$ Research and Development Unit, National Heart Centre, Singapore, Singapore

\section{Correspondence to} Dr Reginald Liew, Consultant Cardiologist, National Heart Centre Singapore, 17 Third Hospital Avenue, Singapore 168752, Singapore; reginald. liew.k.c@nhcs.com.sg

Received 30 October 2011 Revised 8 December 2011 Accepted 9 December 2011 Published Online First 27 January 2012
This paper is freely available online under the BMJ Journals unlocked scheme, see http:// heart.bmj.com/site/about/ unlocked.xhtml

\section{ABSTRACT}

The emergence of induced pluripotent stem cell (iPSC) technology has had a great impact on the field of medicine ever since the ground-breaking discovery in 2006 that overexpression of four specific transcription factors was able to turn back the developmental clock of somatic cells into an embryonic-like state. The resulting iPSCs carry the developmental potential of human embryonic stem cells (hESC) without the embryo and have been heralded as a powerful tool to study development and disease. This technology has made it possible for the first time for researchers to transform end-differentiated cells from a particular individual into another cell type that remains specific to that individual, paving the way for novel methods of in vitro disease modelling and therapeutic applications. This paper reviews some of the key areas in cardiovascular medicine in which iPSC technology has been applied and discusses the future directions and ongoing challenges ahead in this exciting field.

\section{INTRODUCTION}

The emergence of induced pluripotent stem cell (iPSC) technology has had a great impact on the field of medicine ever since ground-breaking work by Yamanaka and colleagues demonstrated that the overexpression of four genes (or 'Yamanaka factors': Oct4, Sox2, Klf4, c-Myc) was able to turn back the developmental clock of somatic cells into an embryonic-like state. ${ }^{1}$ The resulting iPSCs carry the developmental potential of human embryonic stem cells (hESC) without the embryo and have been heralded as a powerful tool to study development and disease. The importance of Yamanaka's pioneering work is that, for the first time, researchers have a tool to transform end-differentiated cells from a particular individual into another cell type that remains specific to that individual while, at the same time, circumventing many of the ethical dilemmas that have hampered traditional embryonic stem cell research to date. The past few years have witnessed an exponential increase in the number of publications on iPSCs, with a number of genetic cardiovascular diseases being successfully modelled in vitro using this technology. ${ }^{3}$ Researchers in this fast-moving field are also moving towards other applications of iPSCs, including regenerative medicine and drug testing and discovery. ${ }^{4}$ There is therefore enormous potential for this new technology, both in a greater understanding of disease mechanisms and improved treatments. Moreover, the fact that the iPSCs are produced from a patient's own tissue means that the results of in vitro testing are directly relevant to that individual, allowing the new era of 'personalised medicine' to become more of a reality. At this 5-year mark after the initial iPSC discovery we revisit what has been achieved to date in relation to cardiovascular medicine, examine the future directions and critically appraise the ongoing issues and challenges ahead that need to be overcome prior to widespread clinical application.

\section{GENERATION OF PATIENT-SPECIFIC IPSCS AND CARDIOMYOCYTES}

Several methods have been employed to reprogramme somatic cells into iPSCs, which can broadly be divided into integrating or non-integrating methods. $^{3}$ Integrating methods involve viral delivery (using retro- or lentiviruses) and integration of the Yamanaka factors into the host genome while non-viral non-integrating methods involve the transient expression of reprogramming factors (eg, episomal plasmid vectors, minicircle vectors, RNA and protein delivery) without genomic integration. In addition, some laboratories have reported success using viral non-integrating methods. Methods that involve genomic integration carry several disadvantages, including random integration into the host genome resulting in the expression of endogenous genes and the development of a tumorigenic phenotype. This may not be a major issue if the iPSCs are used for disease modelling and in vitro testing, but presents a major barrier to use of these cells as a therapeutic option, as will be discussed later. Non-integrating methods are therefore a more suitable option for iPSC-based therapeutic applications. In our laboratory we have successfully reprogrammed patients' dermal fibroblasts into iPSCs using both integrating and nonintegrating methods. A schematic diagram of the stages involved for the generation of patientspecific iPSCs and cardiomyocytes from dermal fibroblasts using the viral integrating method is shown in figure 1 .

iPSC colonies can be differentiated into functional cardiomyocytes using a variety of methods, which are very similar to those traditionally employed to produce cardiomyocytes from hESCs since both iPSCs and hESCs share very similar characteristics and differentiation potential. Currently, the most common method of generating cardiomyocytes from iPSCs is the embryoid body (EB) differentiation system which coaxes the iPSCs to differentiate into the cardiac lineage. ${ }^{3}$ Light microscopic images of iPSC-derived cardiomyocytes (iPSC-CMs) produced from patient dermal fibroblast samples are shown in figure 2 . Although the iPSC-CMs show many of the functional 
Figure 1 Schematic diagram showing the generation of induced pluripotent stem cell-derived cardiomyocytes (iPSC-CMs) from human dermal fibroblasts. Non-embryonic stem celllike colonies which appear first are not picked. Only embryonic stem cell-like colonies which appear later are picked and maintained. MEF, mouse embryonic fibroblast; MEA, microelectrode array.



characteristics of normal human cardiomyocytes (discussed below), some other aspects of these cells, including their morphology, remain relatively immature and 'fetal-like', as can be seen in figure 2.

\section{FUNCTIONAL CHARACTERISATION OF IPSC-DERIVED CARDIOMYOCYTES}

Before iPSC-CMs can be considered for use as disease models or in regenerative medicine, the cells produced need to be confirmed to exhibit the essential functional characteristics of human cardiomyocytes. The cellular phenotype of iPSC-CMs can be characterised using several methods including electrophysiological and molecular biological techniques. Whole cell patch clamping can be performed on individual cardiomyocytes after cells are dissociated from the EBs, allowing for measurement of specific ionic currents and action potential (AP) profiles. Three types of AP morphologies can usually be recorded from iPSCCMs: 'atrial-like', 'ventricular-like' and 'nodal-like' morphologies (figure $3 \mathrm{~A}$ ). In our experience, the majority of cardiomyocytes produced using viral-based methods of reprogramming (approximately 65\%) exhibit a ventricular-like AP profile. The iPSC-CMs appear to respond to known drugs affecting the AP in the expected way-for example, the AP is prolonged in the presence of the delayed recitifier potassium current $\left(\mathrm{I}_{\mathrm{Kr}}\right)$ blocker E-4031 (figure 3C). Electrophysiological properties of the iPSCCMs can also be evaluated at the multicellular level using microelectrode array mapping techniques; areas within EBs observed to be contracting are dissected and placed on microelectrode array plates where field potentials and morphologies across the cells of interest can be recorded. Traditional methods of assessing intracellular $\mathrm{Ca}^{2+}$ handling, such as use of $\mathrm{Ca}^{2+}$-sensitive fluorescent indicators and confocal microscopy, can be applied to iPSC-CMs to confirm the presence of intact and functional $\mathrm{Ca}^{2+}$ handling apparatus (figure 3D). Molecular biological techniques to confirm functionality of the iPSC-CMs include immunocytochemistry, phosphoproteomic assays and quantitative reverse transcriptase PCR (qRT-PCR) to identify molecular targets and expression levels of key proteins found in normal functional cardiomyocytes.
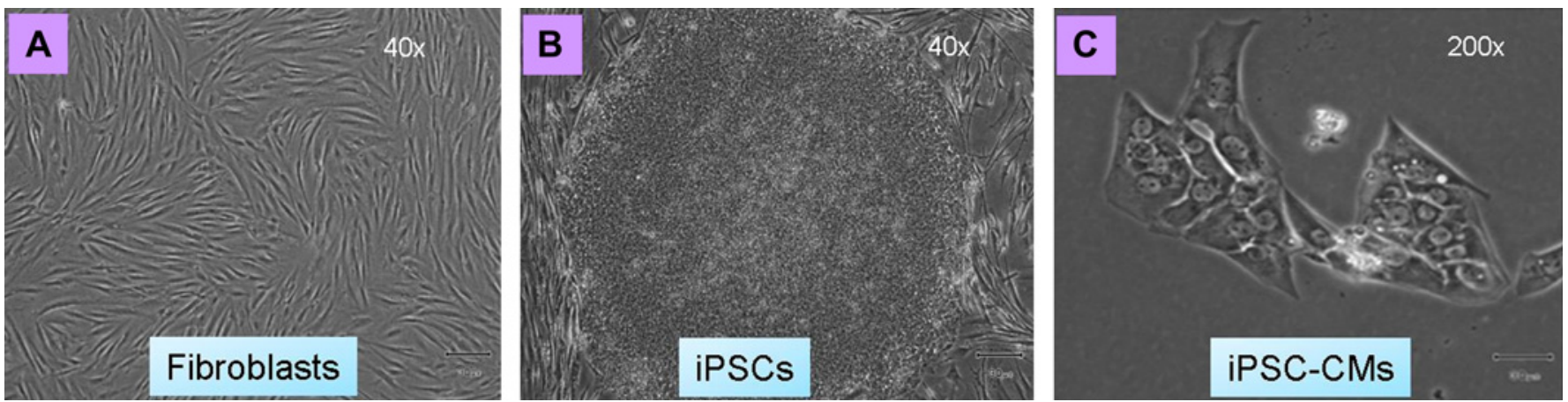

Figure 2 Light microscopic images of typical dermal fibroblasts, human induced pluripotent stem cells (iPSCs) and iPSC-derived cardiomyocytes (iPSC-CMs). (A) Parental human dermal fibroblasts (FB) in culture (magnification $\times 40$ ). (B) Colony of human iPSCs derived from dermal fibroblasts via retroviral-based reprogramming using Yamanaka factors (magnification $\times 40$ ). (C) Small cluster of contracting cardiomyocytes (iPSC-CMs) dissociated from the contracting embryoid bodies derived from iPSCs (magnification $\times 200$ ). 
A

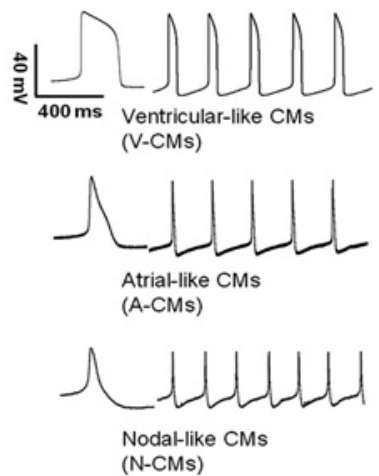

B

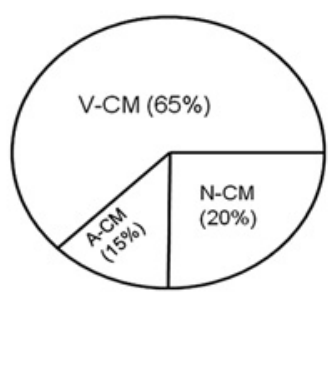

C


D

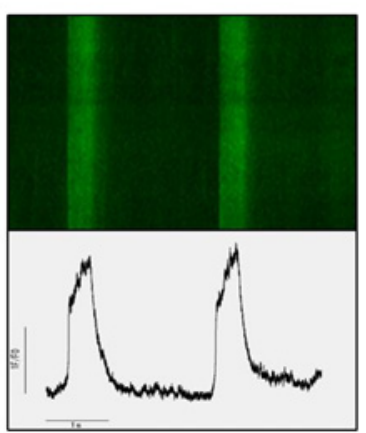

Figure 3 Electrophysiological characterisation of human induced pluripotent stem cell-derived cardiomyocytes (iPSC-CMs). (A) Action potential traces of three subtypes of human iPSC-CMs determined by patch clamp. (B) Relative proportion of three subtypes of cardiomyocytes (ventricular CMs (V-CMs), atrial CMs (A-CMs) and nodal-CMs (N-CMs)) randomly selected from 25 cardiomyocytes differentiated from a line of iPSCs. (C) Change in action potential (AP) profile in the presence of $5 \mu \mathrm{mol}$ of the $\mathrm{I}_{\mathrm{kr}}$ blocker E-4031. E-4031 increased the AP duration due to the prolongation of phase 3 of the AP. (D) $\mathrm{Ca}^{2+}$ transients recorded from iPSC-CMs using confocal microscopy (top) and $\mathrm{Ca}^{2+}$ transient profiles (bottom).

\section{CARDIOVASCULAR DISEASE MODELLING}

\section{Advantage of iPSCs over hESCs in disease modelling}

Although both hESCs and iPSCs share the pluripotent state and common utility in disease modelling and therapy, the adult does not have any hESCs. On the other hand, not only does the derivation of iPSCs from human somatic cells circumvent the ethical roadblock associated with the acquisition of hESCs, it is a procedure which has been achieved by a number of independent laboratories, including ours, using a standard and relatively straightforward protocol. In contrast to hESC-CMs, iPSC-CMs derived from somatic cells of a particular patient have the distinct advantage that the cells are individualised, allowing the study of a genetic disease model that is unique to the patient. This has paved the way for iPSC-based technology to be used in human disease modelling, including investigation of rarer diseases. ${ }^{6-8}$

A good starting point to investigate the usefulness of iPSC technology in modelling human cardiovascular diseases is to study certain inherited arrhythmogenic diseases caused by relatively simple mutations in which a clear relationship between genotype and phenotype can be demonstrated. Since the patient-specific iPSC-CMs should harbour the same diseasecausing mutation as is present in the host tissue (skin fibroblasts or hair follicle), researchers could in theory reproduce the clinical phenotype 'in the petri dish' and thus establish an in vitro model of the disease. A number of pioneering proof-of-concept studies have been published over the past year in patients with inherited arrhythmogenic diseases, most notably different subtypes of long OT syndrome, which confirm that patient-specific iPSCCMs can indeed recapitulate the clinical phenotype in vitro and thus represent a potential and exciting new method for disease modelling. These models will allow researchers to better understand disease mechanisms as well as to investigate the effects of different compounds on the cellular electrophysiology of the cells, allowing for novel drug discovery or treatments. Table 1 summarises the studies in humans to date for which iPSC technology has been used to generate in vitro models of a number of cardiovascular diseases.

\section{Modelling the long OT syndrome}

Moretti et al were the first to show that iPSC-CMs can recapitulate the disease phenotype in humans. ${ }^{9}$ They identified an autosomal dominant inheritance of a $596 \mathrm{G} \rightarrow \mathrm{A}$ missense mutation in the KCNO1 gene within a family which has previously been shown to be associated with long OT syndrome type 1 (LOTS1). Electrophysiological parameters in iPSC-CMs generated from two patients with LOTS1 were compared with those generated from two unrelated healthy control subjects using whole-cell patch clamping. AP duration was found to be significantly prolonged and the repolarisation velocity reduced in LOTS1 iPSC-CMs compared with controls. Furthermore, single cell electrophysiological analysis showed a reduction specifically in the $\mathrm{I}_{\mathrm{KS}}$ current in ventricular LOTS1 iPSC-CMs compared with controls with no significant differences in the other currents measured.

Similarly, two other groups reported their findings on the use of iPSC-CM disease models for LQTS2. Itzhaki et al obtained dermal fibroblasts from a patient with LOTS2 harbouring a known KCNH2 gene mutation (affecting $\mathrm{I}_{\mathrm{Kr}}$ ) and showed that AP duration was prolonged and repolarisation velocity reduced in LOTS2 iPSC-CMs compared with controls. ${ }^{10}$ They measured and verified that $\mathrm{I}_{\mathrm{Kr}}$ was significantly reduced in LOTS2 iPSCCMs. The investigators further tested drugs that could either relieve or aggravate the clinical phenotype of LOTS2 and found AP duration to be prolonged with E-4031, which was associated with new early after depolarisations (EADs) or an increase in the number or complexity of EADs. The potential therapeutic effects of nifedipine and the $\mathrm{K}_{\mathrm{ATP}}$ channel opener pinacidil (which augments the outward potassium current) was tested in this in vitro model; the drugs were demonstrated to shorten the AP duration and abolish EADs. Matsa et al also successfully generated iPSC-CMs from a patient with LOTS2 with a known KCNH2 mutation and her mother, a carrier for the same mutation. ${ }^{11}$ LOTS2 iPSC-CMs displayed prolonged AP duration on patch clamp analysis and also prolonged corrected field potential duration (cFPD) on microelectrode array mapping, in concordance with the results of Itzhaki et al. Similarly, Matsa et al showed that application of E-4031 prolonged the AP duration and induced EADs. In addition, they tested the effect of the $\mathrm{K}_{\mathrm{ATP}}$ channel opener nicorandil and PD-118057, a type $2 \mathrm{I}_{\mathrm{Kr}}$ channel enhancer that attenuates channel closing, on LOTS2 iPSC-CMs. Both were able to shorten the AP duration when used alone or in combination. In addition, nicoranidil was able to abolish EADs.

Yazawa et al generated iPSC-CMs from two patients with Timothy syndrome (LOTS type 8 ) in which a mutation in the CACNA1C gene which encodes the Cav1.2 channel in humans leads to a disorder characterised by LOTS, immunodeficiency, 


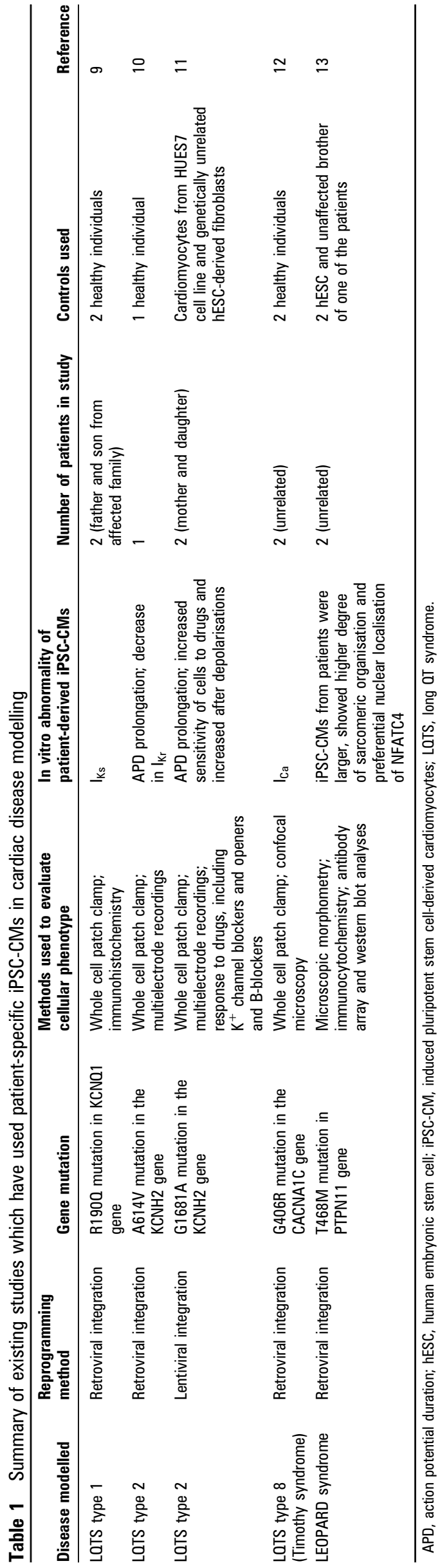

syndactyly and autism. ${ }^{12}$ They analysed time-lapse images of spontaneously contracting EBs and found that Timothy syndrome EBs contracted at a slower rate and more irregularly than control EBs. They also found that the L-type channel current in iPSC-CMs derived from the patients with Timothy syndrome had significantly reduced voltage-dependent inactivation compared with control cells. Only ventricular-like iPSCCMs from patients with Timothy syndrome had APs that were significantly prolonged compared with controls, while atrial and nodal Timothy syndrome iPSC-CMs did not. This is in contrast to the study by Moretti et al on LOTS1 iPSC-CMs in which both ventricular and atrial-like iPSC-CMs displayed the disease phenotype. In addition, Yazawa et al observed that arrhythmias and delayed depolarisations were already evident in spontaneously beating Timothy syndrome iPSC-CMs while arrhythmias were only elicited under condition of adrenergic stress with the application of isoproterenol in LOTS1. Given that Cav1.2 plays a role in $\mathrm{Ca}^{2+}$-induced $\mathrm{Ca}^{2+}$ release from the sarcoplasmic reticulum, the investigators demonstrated that Cav1.2 inactivation in Timothy syndrome could affect the timing and amplitude of ventricular $\mathrm{Ca}^{2+}$ release, leading to larger and more prolonged $\mathrm{Ca}^{2+}$ elevations.

\section{Modelling other inherited cardiovascular diseases}

In theory, other cardiovascular diseases with a hereditary component may be modelled using iPSC technology providing the iPSC-CMs display the key phenotypic features associated with the disease of interest. However, the process becomes more complex and difficult with increased heterogeneity of the disease (both phenotypically and genetically) or with greater involvement of external factors during the natural history of the disease. LEOPARD syndrome, an autosomal dominant developmental disorder in which the PTPN11 gene encoding a SHP2 phophatase is mutated, and the associated hypertrophic cardiomyopathy (HCM) phenotype of the disease has been modelled successfully using iPSC technology. ${ }^{13}$ The investigators found that iPSC-CMs generated from two patients with LEOPARD syndrome had a larger median surface area, a greater degree of sarcomeric assembly and a greater degree of NFAT nuclear localisation than iPSC-CMs from control patients without disease. Protein molecular targets that could be potentially implicated in the LEOPARD syndrome phenotype were identified through proteomic assays, and certain proteins were found to be more abundant or had increased phosphorylation in cardiomyocytes derived from the patients with LEOPARD syndrome compared with control subjects. Our group has successfully generated iPSC-CMs from a patient with arrhythmogenic right ventricular cardiomyopathy (ARVC) harbouring a PKP2 mutation and are in the process of modelling the disease in vitro. ${ }^{14}$ However, significant hurdles still exist in modelling the more complex cardiovascular diseases using iPSC technology. These include: (1) difficulty in ensuring a purified cardiomyocyte population from iPSCs through standard cardiomyocyte differentiation protocols; (2) the complexities of reproducing a heterogeneous disease phenotype (eg, fibrofatty change and increased arrhythmogenicity in ARVC, myofibril disarray in HCM) which may involve other systemic factors in vitro using only cardiomyocytes; and (3) limitations of modelling essentially adult-onset diseases (for certain types of cardiomyopathies) using iPSC-CMs with a predominantly fetal-like phenotype. It is likely that further in vitro manipulation of iPSC-CMs will be required to model the more complex diseases successfully. This may involve measures such as overexpression of apolipoprotein-A1 cDNA or enhancement of the 
Wnt/ $\beta$-catenin signalling pathway in order to generate a more mature phenotype. ${ }^{1516}$ Another promising avenue appears to be in the development of novel methods of engineering cardiac tissue from iPSC-CMs ${ }^{17}$ and assessment of in vitro contractility. ${ }^{18}$ Until such methods are optimised and further research is performed in the area of maturation of iPSC-CMs, modelling of the more complex cardiovascular diseases including the cardiomyopathies will remain a major challenge.

\section{APPLICATIONS IN DRUG TESTING AND DISCOVERY In vitro drug screening}

Part of the process of drug development and testing of new products for clinical use includes the demonstration that the product does not have any significant cardiac toxicities or proarrhythmic actions. Early and accurate detection of potential cardiac toxicities could save the pharmaceutical industry millions of dollars, avoiding additional funds being wasted to develop the drug further. There is an increasing demand for good human models of healthy as well as diseased hearts, given the limitations of current animal models that are currently used in drug testing and fundamental differences in cardiac electrophysiology between animals and humans. Recent studies have demonstrated that hESC-CMs may represent a novel platform for electrophysiological drug screening as the cells respond to a number of cardiac and non-cardiac drugs in the predicted way. ${ }^{19} 20$

\section{Applications in personalised medicine}

As demonstrated from the human cardiac disease models generated so far, several drugs have been tested on patientspecific iPSC-CMs and found either to ameliorate or alleviate the disease phenotype. ${ }^{9-11}$ The iPSC-CM disease model allows the concept of 'personalised medicine' to be put forward as these cells will in theory provide information on the effects of a particular drug on the individual from which the cells were obtained. Thus, using this technology, clinicians and scientists may be able to ascertain the cardiac response or side effects of an individual to a new drug in vitro before the drug is actually tested out in the individual. Further work needs to be done before this application can be realised, including evidence that the iPSC-CMs generated faithfully reproduce the clinical phenotype and response to drugs in vitro (in both normal and diseased individuals) and improved methods of iPSC-CM generation, since current methods are too laborious and timeconsuming for routine clinical application. However, this remains an exciting and novel application of iPSC technology which is not possible with other currently available methods.

Another promising future application of iPSC technology is the concept of targeted gene modification of patient-specific iPSCs using homologous recombination techniques and zinc finger nucleases (ZFNs) with the exciting prospect of correcting disease-causing mutations. ${ }^{21}$ This strategy has several potential advantages over conventional gene therapy, including avoidance of the need for immunosuppression and elimination of the risk of insertional mutagenesis caused by therapeutic vectors. The technique has recently been successfully applied in iPSCs to correct the genetic mutations responsible for $\alpha_{1}$-antitrypsin deficiency ${ }^{22}$ and sickle cell anaemia, ${ }^{23}$ although it is still currently in its infancy and thus remains challenging and inefficient.

\section{Applications in regenerative medicine Myocardial repair}

To date, a wide variety of different stem cell types have been investigated for their ability to repair the infarcted heart in both animal studies and human clinical trials. ${ }^{24}$ The emergence of iPSC technology adds an additional cell source that may have a potential clinical application in this area, although research in regenerative medicine using these cells is still at an early stage. ${ }^{25}$ The major advantage of iPSCs over other cell types for cell transplantation is the fact that these cells are patient-specific, thereby circumventing the important issue of tissue rejection associated with transplantation procedures. However, enthusiasm for use of iPSC technology in regenerative medicine has been hampered by the increasingly recognised problems of the unpredictable nature and behaviour of transplanted iPSCs and the potential for these cells to cause harm. ${ }^{26}$ Additional issues such as the potential teratogenicity of undifferentiated cells and proarrhythmia of iPSC-CMs need to be addressed before clinical grade iPSCs can be used in regenerative medicine.

To date, several disease models such as sickle cell anaemia, ${ }^{27}$ Parkinson's disease ${ }^{28}$ and haemophilia $\mathrm{A}^{29}$ have been successfully treated using iPSC-based therapy. In relation to cardiovascular medicine, Nelson et al showed that intramyocardial delivery of iPSCs into the infarcted hearts of mice following ligation of the left anterior descending artery yielded progeny that properly engrafted without disrupting cytoarchitecture. ${ }^{30}$ Furthermore, the investigators found that iPSC treatment restored contractile performance, ventricular wall thickness and electrical stability, while also achieving in situ regeneration of cardiac, smooth muscle and endothelial tissue. In another proof-of-concept study, Mauritz et al showed that injection of iPSC-derived fetal liver kinase-1 (Flk-1) positive progenitor cells into the ischaemic myocardium of left anterior descending artery-ligated mice improved cardiac function following myocardial infarction and that the Flk-1 positive cells differentiated into cardiovascular lineages in vitro and in vivo. ${ }^{31}$

\section{Biological pacemakers}

Another potential and exciting application of iPSC technology in cardiovascular medicine is in the development of cell-based therapies for biological pacing. The advent of electronic pacemakers has transformed the treatment and lives of many patients suffering from symptomatic bradyarrhythmias over the past few decades. However, a number of important limitations still exist with permanent pacemakers, including the need for a surgical procedure and its associated risks, the ongoing risk of infection and the need for frequent generator changes and possible lead changes over the lifetime of the individual (this becomes more of an issue the younger the patient is at first implant). The concept of a 'biological pacemaker' which can integrate with the host cardiac tissue, generate the electrical impulses required by the individual (whose intrinsic pacemaker function is defective/inadequate) and physiologically adapt to the cardiac requirements of that individual is certainly an attractive one. One of the first major reports on the use of stem cells for biological pacing involved the implantation of cardiomyocytes derived from hESCs into the ventricles of pigs with complete heart block. ${ }^{32}$ The investigators performed histological examination and detailed 3D-electroanatomical mapping to show that the hESC-CMs integrated with the host cardiac tissue and were able to pace the swine hearts at a higher rate than the back-up rate set by the electronic pacemaker. Similar findings were obtained by another group using genetically engineered hESCs transplanted into guinea pig hearts. ${ }^{33}$ This approach, however, requires immunosuppression if applied to humans since the hESCs are not obtained from the host. The use of patient-specific iPSC-CMs may overcome this problem, although other potential issues regarding tumorigenicity, 
Table 2 Summary of current and future applications of iPSC technology in relation to cardiovascular medicine and ongoing challenges ahead

\begin{tabular}{|c|c|c|}
\hline Cardiovascular application & Advantage of using iPSC-technology & Ongoing challenges \\
\hline \multicolumn{3}{|l|}{ Disease modelling } \\
\hline $\begin{array}{l}\text { Inherited arrhythmogenic diseases } \\
\text { - Inherited cardiomyopathies }\end{array}$ & $\begin{array}{l}\text { iPSC-CMs carry same genetic } \\
\text { mutation allowing rarer genetic } \\
\text { diseases to be modelled }\end{array}$ & $\begin{array}{l}\text { Relatively immature phenotype } \\
\text { Difficulties in modelling more complex } \\
\text { multifactorial diseases }\end{array}$ \\
\hline \multicolumn{3}{|l|}{ Drug testing and discovery } \\
\hline $\begin{array}{l}\text { Testing of existing drugs on human } \\
\text { cardiomyocytes } \\
\text { Testing of new drugs } \\
\text { Personalised medicine }\end{array}$ & $\begin{array}{l}\text { Unlimited source of human } \\
\text { cardiomyocytes }\end{array}$ & $\begin{array}{l}\text { Relatively immature phenotype } \\
\text { Need to improve efficiency of iPSC-CM } \\
\text { production and upscaling of cells }\end{array}$ \\
\hline $\begin{array}{l}\text { In vitro drug testing } \\
\text { - Correction of genetic mutation }\end{array}$ & $\begin{array}{l}\text { Patient-specific iPSC-CMs: in vitro } \\
\text { data may be directly relevant to } \\
\text { individual } \\
\text { Possibility of correcting genetic } \\
\text { mutation specific to that individual }\end{array}$ & $\begin{array}{l}\text { Need to demonstrate good correlation } \\
\text { between in vitro data and significance } \\
\text { to individual } \\
\text { Current methods of reprogramming too } \\
\text { slow and inefficient }\end{array}$ \\
\hline \multicolumn{3}{|l|}{ Regenerative medicine } \\
\hline $\begin{array}{l}\text { Cell transplantation in heart failure/ } \\
\text { post myocardial infarction } \\
\text { Biological pacemakers } \\
\text { Artificial heart valves } \\
\text { Vascular endothelial cells for } \\
\text { peripheral vascular disease }\end{array}$ & $\begin{array}{l}\text { No need for immunosuppression } \\
\text { iPSC-CMs can be produced from } \\
\text { patients' own somatic cells }\end{array}$ & $\begin{array}{l}\text { Potential teratoma formation of } \\
\text { undifferentiated iPSCs } \\
\text { Potential proarrhythmias } \\
\text { Punctional and electrical integration } \\
\text { of cells } \\
\text { Need for purification and upscaling } \\
\text { of iPSC-CMs } \\
\text { Optimal delivery methods }\end{array}$ \\
\hline
\end{tabular}

iPSC-CM, induced pluripotent stem cell-derived cardiomyocytes.

requirement for functional and electrical integration with host tissue and methods of cell delivery remain.

\section{Ongoing challenges for use of iPSC technology in regenerative medicine}

Despite the encouraging results from preclinical studies, a number of obstacles still need to be overcome before iPSCbased therapy can be applied to humans for therapeutic purposes. One of the most important of these is the potential of transplanted undifferentiated iPSCs to form teratomas. Consequently, undifferentiated cells should be removed from the grafts prior to transplantation. This may be achieved by manual dissection of areas of beating cardiomyocytes or by using fluorescence-activated cell sorting with specific dyes to select out a high purity of cardiomyocytes. ${ }^{34} 35$ Other ways to circumvent this issue may involve the generation of partially induced pluripotent stem cells, which do not appear to form tumours in vivo and can also engraft into the heart, ${ }^{36}$ or direct reprogramming of fibroblasts into differentiated cardiomyocytes without involving a pluripotent intermediate. ${ }^{37} 38$ Using stateof-the-art genomic technologies, several investigators have recently provided evidence that in vitro reprogramming and expansion of iPSCs can result in a number of genetic and epigenetic abnormalities. ${ }^{26}$ These include an increase in the number of copy number variations which are not found in cells of origin, deletion or point mutations and abnormalities in DNA methylation status. A greater understanding of these cells and the effects of reprogramming is therefore required before clinical application in regenerative medicine. Another safety concern in relation to the use of stem cells pertains to the potential proarrhythmic effects of transplanted stem cells in the host heart. ${ }^{39} 40$ Other issues include the need to generate and select out large quantities of high purity cardiomyocytes, safe and effective methods of cell delivery and ensuring that transplanted cells remain in the myocardium. Novel transplantation technologies such as cardiospheres (cardiac cell aggregates) ${ }^{41}$ and cardiac cell sheets using temperature-responsive culture surfaces may provide solutions to some of these issues. $^{42}$

\section{CONCLUSIONS}

Differentiation of human iPSCs in vitro into functional cardiomyocytes is now becoming a process which can be effectively manipulated and directed. New and improved protocols for cardiomyocyte differentiation of iPSCs are rapidly accumulating in the literature. ${ }^{43} 44$ Generation of other cell types related to the heart and cardiovascular system using iPSC technology is still in its infancy, although preclinical proof-of-concept studies have shown some potential for the use of such cells in the treatment of peripheral vascular disease ${ }^{45}$ and valvular heart diseases. ${ }^{46}$ Table 2 summarises the current and future applications of iPSC technology in relation to cardiovascular medicine and the ongoing challenges ahead. In the immediate future the most promising application of iPSCs in cardiovascular medicine is in the areas of disease modelling and in vitro drug testing. However, an important issue for researchers to address is in designing strategies that will allow iPSC-CMs to become more mature in vitro and thus more applicable to human disease modelling. Other challenges, particularly in relation to the potential use of iPSCs in regenerative medicine, include the issues of teratogenicity and potential proarrhythmias. In addition, current methods of iPSC reprogramming and differentiation into functional cardiomyocytes are still relatively slow, labour intensive and inefficient. In our laboratory, for example, it still takes approximately 2-4 months to transform somatic human cells into iPSC-CMs and requires dedicated personnel to work manually on the cell lines. Consequently, use of iPSC technology in most specialised research laboratories around the world is still currently in the realms of academic research and discovery. However, improved and faster techniques for generating human iPSC lines are constantly emerging, such as the positive selection of cells expressing specific pluripotency markers ${ }^{47}$ and the use of newer small molecules. ${ }^{48}$ In parallel with these advances, involvement of industry collaborators is also increasing, making commercial and large-scale production of human iPSC-CMs that can be used clinically for drug testing and therapeutics a fast-approaching reality.

Funding Goh Foundation.

Competing interests None. 
Contributors All co-authors contributed to the writing and planning of this review article and to the original figures used from our own data in our research unit.

Provenance and peer review Not commissioned; externally peer reviewed.

\section{REFERENCES}

1. Takahashi $\mathbf{K}$, Yamanaka S. Induction of pluripotent stem cells from mouse embryonic and adult fibroblast cultures by defined factors. Cell 2006;126:663-76.

2. Freund C, Mummery CL. Prospects for pluripotent stem cell-derived cardiomyocytes in cardiac cell therapy and as disease models. J Cell Biochem 2009;107:592-9.

3. Narsinh K, Narsinh KH, Wu JC. Derivation of human induced pluripotent stem cells for cardiovascular disease modeling. Circ Res 2011;108:1146-56

4. Wu SM, Hochedlinger K. Harnessing the potential of induced pluripotent stem cells for regenerative medicine. Nat Cell Biol 2011;13:497-505.

5. Inoue $\mathbf{H}$, Yamanaka $S$. The use of induced pluripotent stem cells in drug development. Clin Pharmacol Ther 2011:89:655-61.

6. Ebert AD, Yu J, Rose FF Jr, et al. Induced pluripotent stem cells from a spinal muscular atrophy patient. Nature 2009;457:277-80.

7. Dimos JT, Rodolfa KT, Niakan KK, et al. Induced pluripotent stem cells generated from patients with ALS can be differentiated into motor neurons. Science 2008:321:1218-21.

8. Liu GH, Barkho BZ, Ruiz S, et al. Recapitulation of premature ageing with iPSCs from Hutchinson-Gilford progeria syndrome. Nature 2011;472:221-5.

9. Moretti A, Bellin M, Welling A, et al. Patient-specific induced pluripotent stem-cell models for long-0T syndrome. N Engl J Med 2010;363:1397-409.

10. Itzhaki I, Maizels L, Huber I, et al. Modelling the long OT syndrome with induced pluripotent stem cells. Nature 2011:471:225-9.

11. Matsa E, Rajamohan D, Dick E, et al. Drug evaluation in cardiomyocytes derived from human induced pluripotent stem cells carrying a long OT syndrome type 2 mutation. Eur Heart J 2011;32:952-62.

12. Yazawa $\mathbf{M}$, Hsueh $\mathrm{B}$, Jia $\mathrm{X}$, et al. Using induced pluripotent stem cells to investigate cardiac phenotypes in Timothy syndrome. Nature 2011;471:230-4.

13. Carvajal-Vergara $\mathbf{X}$, Sevilla A, D'Souza SL, et al. Patient-specific induced pluripotent stem-cell-derived models of LEOPARD syndrome. Nature 2010:465:808-12

14. Ma D, Wei H, Guangqin Z, et al. Generation of induced pluripotent stem cells and cardiomyocytes as a cellular model of arrhythmogenic right ventricular cardiomyopathy [abstract]. Circulation 2011:124:21. Supplement (A12906).

15. $\mathbf{N g ~ K M , ~ L e e ~ Y K , ~ L a i ~ W H , ~ e t ~ a l . ~ E x o g e n o u s ~ e x p r e s s i o n ~ o f ~ h u m a n ~ a p o A - I ~ e n h a n c e s ~}$ cardiac differentiation of pluripotent stem cells. PLoS One 2011;6:e19787.

16. Paige SL, Osugi T, Afanasiev OK, et al. Endogenous Wnt/beta-catenin signaling is required for cardiac differentiation in human embryonic stem cells. PLoS One 2010;5 e11134.

17. Sarig U, Machluf M. Engineering cell platforms for myocardial regeneration. Expert Opin Biol Ther 2011:11:1055-77.

18. Alford PW, Feinberg AW, Sheehy SP, et al. Biohybrid thin films for measuring contractility in engineered cardiovascular muscle. Biomaterials 2010;31:3613-21.

19. Braam SR, Tertoolen L, van de Stolpe A, et al. Prediction of drug-induced cardiotoxicity using human embryonic stem cell-derived cardiomyocytes. Stem Cell Res 2010;4:107-16

20. Caspi 0, Itzhaki I, Kehat I, et al. In vitro electrophysiological drug testing using human embryonic stem cell derived cardiomyocytes. Stem Cells Dev 2009;18:161-72.

21. Collin J, Lako M. Concise review: putting a finger on stem cell biology: zinc finger nuclease-driven targeted genetic editing in human pluripotent stem cells. Stem Cells 2011;29:1021-33

22. Yusa K, Rashid ST, Strick-Marchand H, et al. Targeted gene correction of alpha1antitrypsin deficiency in induced pluripotent stem cells. Nature 2011;478:391-4.

23. Sebastiano V, Maeder ML, Angstman JF, et al. In situ genetic correction of the sickle cell anemia mutation in human induced pluripotent stem cells using engineered zinc finger nucleases. Stem Cells 2011;29:1717-26.
24. Lovell MJ, Mathur A. Cardiac stem cell therapy: progress from the bench to bedside. Heart 2010;96:1531-7.

25. Yoshida Y, Yamanaka S. iPS cells: a source of cardiac regeneration. J Mol Cell Cardiol 2011:50:327-32.

26. Pera MF. Stem cells: the dark side of induced pluripotency. Nature 2011;471:46-7

27. Hanna J, Wernig M, Markoulaki S, et al. Treatment of sickle cell anemia mouse model with iPS cells generated from autologous skin. Science 2007;318:1920-3.

28. Wernig M, Zhao JP, Pruszak J, et al. Neurons derived from reprogrammed fibroblasts functionally integrate into the fetal brain and improve symptoms of rats with Parkinson's disease. Proc Natl Acad Sci U S A 2008;105:5856-61.

29. Xu D, Alipio Z, Fink LM, et al. Phenotypic correction of murine hemophilia A using an iPS cell-based therapy. Proc Natl Acad Sci U S A 2009;106:808-13.

30. Nelson TJ, Martinez-Fernandez A, Yamada S, et al. Repair of acute myocardial infarction by human stemness factors induced pluripotent stem cells. Circulation 2009:120:408-16.

31. Mauritz C, Martens A, Rojas SV, et al. Induced pluripotent stem cell (iPSC)-derived Flk-1 progenitor cells engraft, differentiate, and improve heart function in a mouse model of acute myocardial infarction. Eur Heart J 2011;32:2634-41.

32. Kehat I, Khimovich L, Caspi 0, et al. Electromechanical integration of cardiomyocytes derived from human embryonic stem cells. Nat Biotechnol 2004;22:1282-9.

33. Xue T, Cho HC, Akar FG, et al. Functional integration of electrically active cardiac derivatives from genetically engineered human embryonic stem cells with quiescen recipient ventricular cardiomyocytes: insights into the development of cell-based pacemakers. Circulation 2005;111:11-20.

34. Huber I, Itzhaki I, Caspi 0 , et al. Identification and selection of cardiomyocytes during human embryonic stem cell differentiation. FASEB J 2007;21:2551-63.

35. Hattori F, Chen $\mathrm{H}$, Yamashita $\mathrm{H}$, et al. Nongenetic method for purifying stem cellderived cardiomyocytes. Nat Methods 2010:7:61-6.

36. Margariti A, Winkler B, Karamariti E, et al. Direct reprogramming fibroblasts into endothelial cells [abstract]. Heart 2011;97:e7.

37. Efe JA, Hilcove S, Kim J, et al. Conversion of mouse fibroblasts into cardiomyocytes using a direct reprogramming strategy. Nat Cell Biol 2011;13:215-22.

38. Ieda $\mathbf{M}, \mathrm{Fu}$ JD, Delgado-Olguin $\mathrm{P}$, et al. Direct reprogramming of fibroblasts into functional cardiomyocytes by defined factors. Cell 2010;142:375-86.

39. Liao SY, Liu Y, Siu CW, et al. Proarrhythmic risk of embryonic stem cell-derived cardiomyocyte transplantation in infarcted myocardium. Heart Rhythm 2010; 7:1852-9.

40. Menasche $\mathbf{P}$. Stem cell therapy for heart failure: are arrhythmias a real safety concern? Circulation 2009:119:2735-40.

41. Johnston PV, Sasano T, Mills K, et al. Engraftment, differentiation, and functional benefits of autologous cardiosphere-derived cells in porcine ischemic cardiomyopathy. Circulation 2009;120:1075-83, 7.

42. Shimizu T, Sekine H, Yamato M, et al. Cell sheet-based myocardial tissue engineering: new hope for damaged heart rescue. Curr Pharm Des 2009:15:2807-14.

43. Burridge PW, Thompson S, Millrod MA, et al. A universal system for highly efficien cardiac differentiation of human induced pluripotent stem cells that eliminates interline variability. PLoS One 2011;6:e18293.

44. Fujiwara M, Yan P, Otsuji TG, et al. Induction and enhancement of cardiac cell differentiation from mouse and human induced pluripotent stem cells with cyclosporin-A. PLoS One 2011;6:e16734.

45. Rufaihah AJ, Huang NF, Jame S, et al. Endothelial cells derived from human iPSCS increase capillary density and improve perfusion in a mouse model of peripheral arterial disease. Arterioscler Thromb Vasc Biol 2011;31:e72-9.

46. Schmidt D, Dijkman PE, Driessen-Mol A, et al. Minimally-invasive implantation of living tissue engineered heart valves: a comprehensive approach from autologous vascular cells to stem cells. J Am Coll Cardiol 2010;56:510-20.

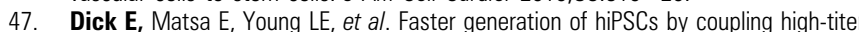
lentivirus and column-based positive selection. Nat Protoc 2011;6:701-14.

48. Zhang Z, Gao Y, Gordon A, et al. Efficient generation of fully reprogrammed human iPS cells via polycistronic retroviral vector and a new cocktail of chemical compounds. PLoS One 2011;6:e26592. 